\title{
Construction of ultrasonic nanobubbles carrying CAIX polypeptides to target carcinoma cells derived from various organs
}

\author{
Lianhua Zhu', Yanli Guo ${ }^{1 *} \mathbb{D}$, Luofu Wang ${ }^{2}$, Xiaozhou Fan', Xingyu Xiong ${ }^{1}$, Kejing Fang ${ }^{1}$ and Dan Xu
}

\begin{abstract}
Background: Ultrasound molecular imaging is a novel diagnostic approach for tumors, whose key link is the construction of targeted ultrasound contrast agents. However, available targeted ultrasound contrast agents for molecular imaging of tumors are only achieving imaging in blood pool or one type tumor. No targeted ultrasound contrast agents have realized targeted ultrasound molecular imaging of tumor parenchymal cells in a variety of solid tumors so far. Carbonic anhydrase IX (CAIX) is highly expressed on cell membranes of various malignant solid tumors, so it's a good target for ultrasound molecular imaging. Here, targeted nanobubbles carrying CAIX polypeptides for targeted binding to a variety of malignant tumors were constructed, and targeted binding ability and ultrasound imaging effect in different types of tumors were evaluated.
\end{abstract}

Results: The mean diameter of lipid targeted nanobubbles was $(503.7 \pm 78.47) \mathrm{nm}$, and the polypeptides evenly distributed on the surfaces of targeted nanobubbles, which possessed the advantages of homogenous particle size, high stability, and good safety. Targeted nanobubbles could gather around CAIX-positive cells (786-O and Hela cells), while they cannot gather around CAIX-negative cells (BXPC-3 cells) in vitro, and the affinity of targeted nanobubbles to CAIX-positive cells were significantly higher than that to CAIX-negative cells $(P<0.05)$. Peak intensity and duration time of targeted nanobubbles and blank nanobubbles were different in CAIX-positive transplanted tumor tissues in vivo ( $P<0.05)$. Moreover, targeted nanobubbles in CAIX-positive transplanted tumor tissues produced higher peak intensity and longer duration time than those in CAIX-negative transplanted tumor tissues $(P<0.05)$. Finally, immunofluorescence not only confirmed targeted nanobubbles could pass through blood vessels to enter in tumor tissue spaces, but also clarified imaging differences of targeted nanobubbles in different types of transplanted tumor tissues.

Conclusions: Targeted nanobubbles carrying CAIX polypeptides can specifically enhance ultrasound imaging in CAIX-positive transplanted tumor tissues and could potentially be used in early diagnosis of a variety of solid tumors derived from various organs.

Keywords: Ultrasound molecular imaging, Targeted nanobubbles, Carbonic anhydrase IX, Malignant tumors

\section{Background}

Early diagnosis and timely treatment of malignant tumors can increase the survival and decrease the morbidity of patients. Compared to contrast-enhanced magnetic resonance imaging (CE-MRI) and contrast-enhanced

\footnotetext{
*Correspondence: guoyanli71@aliyun.com

1 Department of Ultrasound, Southwest Hospital, Third Military Medical University, 30 Gaotanyan Street, Shapingba District, Chongqing 400038, China

Full list of author information is available at the end of the article
}

computed tomography (CE-CT), contrast-enhanced ultrasound (CEUS) has the advantages of easy operation, no radiation and real-time dynamic imaging. Therefore, it has rapidly become popular in clinical application [1]. With the continuous development of CEUS, ultrasound molecular imaging has emerged. Constructing ultrasound contrast agents (UCAs) is the key link in ultrasound molecular imaging. Studies have already confirmed that targeted UCAs carrying specific antibodies or ligands can bind specifically to tissues or disease 
lesions that express specific targets. These agents specifically aggregate in target tissues through the blood circulation, thus achieving targeted ultrasound imaging at the molecular or cellular level [2-4].

Because tumor neovascularization has incomplete basement membranes, lack smooth muscle layer, and blood vessel wall gaps are $380-780 \mathrm{~nm}$, which are much more permeable than vessels in normal tissues, particles smaller than $700 \mathrm{~nm}$ in diameter can theoretically pass through tumor blood vessels and enter into tumor tissue spaces [5]. However, because the size of UCAs currently used is $2-8 \mu \mathrm{m}$, they only can be used for imaging in blood pool and cannot pass through tumor blood vessels to achieve ultrasound molecular imaging of parenchymal cells in tumor tissues [6]. Due to enhanced permeability and retention (EPR) effect, some studies have shown that nanoscale UCAs with the particle size smaller than $700 \mathrm{~nm}$ can be used to achieve targeted ultrasound molecular imaging of tumor parenchyma tissues [7]. Nanobubbles containing a core gas coated with a lipid membrane are a type of nanoscale UCAs. Due to the same contrast enhanced effect as routine clinical UCAs, nanobubbles have broad prospects for clinical application [8-10]. Our previous studies have constructed targeted nanobubbles that specifically target prostate cancer. Imaging duration time and peak intensity provided by these nanobubbles in transplanted tumors were significantly better than those of blank nanobubbles, which could be used for achieving specific targeted imaging of prostate cancer and providing the research basis and methods for targeted molecular imaging of tumors [11-13].

However, our previously constructed targeted nanobubbles had significant limitations. For example, they could only be used in targeted ultrasound imaging of prostate cancer and not in targeted imaging of other tumors, so the tumor type to be selected was limited. Carbonic anhydrase IX (CAIX) is highly expressed on cell membranes of many malignant solid tumors and is not expressed in most normal tissues, except gastrointestinal tracts and biliary tracts. These features are conducive to specific binding between targeted UCAs carrying anti-CAIX ligands and tumor cells and therefore provide an ideal target for ultrasound molecular imaging of a variety of tumors, including kidney cancer and cervical cancer [14-17].

Polypeptides consist of $\alpha$-amino acids linked each other through peptide bonds. The chemical structure of polypeptides is simple, their molecular weight is small, and their properties are stable, so they have been used extensively in medical diagnosis and drug development [1821]. Askoxylakis et al. used the phage display technology to screen polypeptides PGLR-P1 (NMPKDVTTRMSS) against proteoglycan domain of CAIX protein, which is an ideal target ligand of CAIX [22]. Therefore, based on the aforementioned study, we constructed targeted nanobubbles that carry CAIX polypeptides (PGLR-P1) and then tested their safety and relevant physical properties. In addition, the imaging effect of targeted nanobubbles carrying CAIX polypeptides in many CAIX-positive tumors was investigated in vitro and in vivo.

\section{Methods}

Synthesis and validation of the biotinylated polypeptides The biotinylated polypeptides PLGR-P1 (NMPKDVTTRMSSk-biotin) and the biotinylated polypeptides modified with FITC were synthesized using chemical solid-phase synthesis method. The biotinylated polypeptides were synthesized according to the procedures described below: after resin (Nankai Synthesis Technology Co. Ltd, Tianjin, China) swelling by dimethyl formamide (Tianma Medical Fine Chemicals Co. Ltd, Suzhou, China), Fmoc-lysine (Tianma Medical Fine Chemicals Co. Ltd) was incorporated. Then fmoc protection was removed, and amino acids on the resin were detected using ninhydrin (Tianma Medical Fine Chemicals Co. Ltd). Above steps were repeated for each successive amino acid in sequence. At last, the polypeptides were modified with biotin (Tianma Medical Fine Chemicals Co. Ltd). After the synthesis was completed, the synthetic polypeptides were separated and purified by a LC-10ATVP high-performance liquid chromatograph (SHIMADZU, Kyoto, Japan). A LCMS-8030 electrospray ionization mass spectrometer (SHIMADZU) was used to perform mass spectrometry on the pure polypeptides. Some pure biotinylated polypeptides were modified with FITC (Tianma Medical Fine Chemicals Co. Ltd).

\section{Construction and validation of targeted nanobubbles}

A total of $11 \mathrm{mg}$ of 2-dipalmitoyl-sn-glycero-3-phosphocholine (Avanti Polar Lipids, Alabaster, AL, USA), 1,2-dipalmitoyl-sn-glycero-3-phosphoglycerol (Corden Pharma, Liestal, Switzerland), 1,2-dipalmitoyl-snglycero-3-phosphoethanolamine (Corden Pharma), 1,2-dipalmitoyl-sn-glycero-3-phosphatidic acid (Corden Pharma), and biotin-modified 1,2-distearoyl-sn-glycero-3-phosphoethanolamine (NANOCS, Boston, MA, USA) lipid mixture at a certain ratio was dissolved in a mixture solution containing phosphate-buffered saline (PBS) and glycerin mixture, then oscillated overnight in a SHKE420HP orbital shaker (Thermo, Waltham, MA, USA), and transferred to a penicillin bottle. The air in the bottle was replaced with $\mathrm{C}_{3} \mathrm{~F}_{8}$ (Research Institute of Physical and Chemical Engineering of Nuclear Industry, Tianjin, China), and the bottle was oscillated for $80 \mathrm{~s}$ using a ST-B series amalgamator (AT\&M Biomaterials Co., 
Beijing, China). The sample was transferred to a $10-\mathrm{ml}$ centrifuge tube and centrifuged to remove insoluble lipids. After the sample was mixed evenly and centrifuged again, nanobubbles were obtained. Added streptavidin (Solarbio Science \& Technology Co. Ltd, Beijing, China) at a ratio of $3 \mu \mathrm{g}$ streptavidin $/ 10^{7}$ nanobubbles, and the mixture was incubated at $4{ }^{\circ} \mathrm{C}$ for $1 \mathrm{~h}$. After the sample was washed three times, the biotinylated polypeptides were added in a proper ratio, and the mixture was incubated at $4{ }^{\circ} \mathrm{C}$ for $1 \mathrm{~h}$. The mixture was then washed three times, so targeted nanobubbles (TN) carrying CAIX polypeptides were obtained. Blank nanobubbles (BN) that did not carry CAIX polypeptides were used as a control. To directly prove that biotin-streptavidin system could stably and efficiently link the biotinylated polypeptides to the surfaces of targeted nanobubbles, we used the same method to link the FITC-modified biotinylated polypeptides to the surfaces of targeted nanobubbles and used DiI dye (Beyotime Institute of Biotechnology, Shanghai, China) to label targeted nanobubbles lipid membranes, then observed fluorescence intensity of targeted nanobubbles under a Zeiss 780 laser scanning confocal microscope (LSCM) (Carl Zeiss AG, Oberkochen, Germany).

\section{Cell culture in vitro}

Three commonly used cell lines, including 786-O (human kidney carcinoma cells, CAIX-positive cells), Hela (human cervical carcinoma cells, CAIX-positive cells) and BxPC-3 (human pancreatic carcinoma cells, CAIXnegative cells) cells, were used in the study. All cell lines were purchased from the American Type Culture Collection (ATCC, Manassas, VA, USA). The complete medium was made of RPMI 1640 culture medium supplemented with $10 \%$ fetal bovine serum, $100 \mathrm{IU} / \mathrm{ml}$ penicillin, and $100 \mu \mathrm{g} / \mathrm{ml}$ streptomycin. The cells were cultured in an incubator at $37^{\circ} \mathrm{C}$ and in $5 \% \mathrm{CO}_{2}$.

\section{Determination of basic characteristics of targeted nanobubbles}

The concentrations of targeted and blank nanobubbles were measured using a hemocytometer. The particle size and zeta potential of targeted nanobubbles and blank nanobubbles were measured by Malvern Zetasizer nano ZS90 detector (Malvern Instruments Inc., Worcestershire, UK). All measurements were performed three times. The distribution of targeted nanobubbles was observed under an IX71 optical microscope (Olympus Corporation, Kyoto, Japan), and their morphology was observed under a JEM-1400 transmission electron microscope (JEOL, Tokyo, Japan). To evaluate the cytotoxicity of targeted nanobubbles on 786-O cells, MTT assay was performed. 786-O cells at the logarithmic growth phase were inoculated into a 96-well plate at the concentration of $10^{4} /$ well. After culturing overnight, various concentrations of targeted and blank nanobubbles were added. Freshly prepared MTT solution (Beyotime Institute of Biotechnology) was added after the cells had been cultured with nanobubbles for $4 \mathrm{~h}$, then $100 \mu \mathrm{l}$ dimethyl sulphoxide (DMSO) was added. $\mathrm{OD}_{550}$ values were measured using a Multiskan Spectrum (Thermo) to compare the cytotoxicity of targeted and blank nanobubbles. The changes in the concentration and particle size of targeted nanobubbles over time were evaluated. Targeted nanobubbles were kept at $4{ }^{\circ} \mathrm{C}$, and measured at 0 , $1,2,4,7$ days.

\section{In vitro binding experiments with targeted nanobubbles} Logarithmic-phase 786-O, HeLa, and BxPC-3 cells were inoculated into a 24-well plate at the concentration of $10^{5} /$ well. After culturing overnight, the cells were fixed in $4 \%$ paraformaldehyde and blocked in PBS with 5\% bovine serum albumin (BSA-PBS). Each type of cells was divided into three groups. The 1st group received $30 \mu \mathrm{l}$ of targeted nanobubbles at the concentration of $2 \times 10^{7} / \mathrm{ml}$, the 2 nd group received $30 \mu \mathrm{l}$ of the equal concentration of blank nanobubbles, and the 3rd group pre-blocked with $100 \mu \mathrm{l}$ of the polypeptides solution at the concentration of $1 \mathrm{mg} / \mathrm{ml}$ for $2 \mathrm{~h}$ received $30 \mu \mathrm{l}$ of the equal concentration of targeted nanobubbles. Then the cells were incubated with nanobubbles at $4{ }^{\circ} \mathrm{C}$ for $2 \mathrm{~h}$. Slides were washed three times and placed upside down. The number of nanobubbles binding to these three types of cells was observed under an optical microscope. The affinity of targeted nanobubbles to the cells was performed using a FACSCalibur flow cytometer (BD Biosciences, San Jose, CA, USA) [23, 24]. The tumor cells at the logarithmic growth phase were dissociated and collected into Eppendorf tubes at the concentration of $5 \times 10^{5}$ /tube. Each type of cells was divided into three groups. The 1st group served as a control was mixed with only the cells, the 2nd group was mixed the cells with $500 \mu \mathrm{l}$ of blank nanobubbles at the concentration of $2 \times 10^{7} / \mathrm{ml}$, and the 3rd group was mixed the cells with $500 \mu \mathrm{l}$ of the equal concentration of targeted nanobubbles. The mixtures were then incubated at $37^{\circ} \mathrm{C}$ for $40 \mathrm{~min}$. The supernatant was removed after centrifugation. Then the cells were re-suspended in $250 \mu \mathrm{l} \mathrm{PBS}$, and measured the affinity of targeted and blank nanobubbles to the cells by flow cytometry.

\section{In vitro imaging experiments with targeted nanobubbles}

A model for in vitro imaging of targeted nanobubbles was prepared using $1 \%$ agarose powder. IU22 ultrasound diagnosis apparatus and 50-mm linear probe (Philips, Amsterdam, Netherlands) were used for imaging. After the probe was fixed, imaging mode was adjusted (the 
frequency was 5-12 $\mathrm{MHz}$, the mechanical index was 0.12 , and the gain was $85 \%$ ). The imaging intensity of targeted and blank nanobubbles at five different concentrations $\left(1.0 \times 10^{8}, 5.0 \times 10^{7}, 2.5 \times 10^{7}, 1.0 \times 10^{7}\right.$, and $\left.5.0 \times 10^{6} / \mathrm{ml}\right)$ and the decay rate of them at the concentration of $5 \times 10^{7} / \mathrm{ml}$ were compared in vitro. In addition, the imaging intensity of targeted nanobubbles at the concentration of $5 \times 10^{7} / \mathrm{ml}$ was compared before and after destruction by ultrasound waves with the high mechanical index.

\section{In vivo imaging experiments with targeted nanobubbles}

The study was approved by the Laboratory Animal Welfare and Ethics Committee of the Third Military Medical University and was performed in accordance with the NIH Guide for the Care and Use of Laboratory Animals. Logarithmic-phase 786-O and HeLa cells at the concentration of $5 \times 10^{7} / \mathrm{ml}$ and logarithmic-phase BxPC- 3 cells at the concentration of $1 \times 10^{7} / \mathrm{ml}$ were implanted subcutaneously on both sides of 4-week-old BALB/c-nu nude mice back (HFK Bioscience Co., Beijing, China). When the volume of the subcutaneous tumor in tumor-bearing nude mice reached approximately $1 \mathrm{~cm}^{3}$, five 786-O tumor-bearing nude mice, five Hela tumor-bearing nude mice and five BxPC-3 tumor-bearing nude mice were randomly collected and were anesthetized by intraperitoneal injection of $1 \%$ pentobarbital sodium. Ultrasound imaging was performed using IU22 ultrasound diagnosis apparatus and $50-\mathrm{mm}$ linear probe. The surfaces of the transplanted tumors were covered with coupling agents. When the probe clearly displayed the largest cross-section of the transplanted tumor, the probe was fixed. After the 2-dimensional grayscale was collected, the imaging mode was adjusted to contrast-enhanced imaging mode (the frequency was $5-12 \mathrm{MHz}$, the mechanics index was 0.12 , and the gain was $85 \%$ ), and the focus center was located at the center of the transplanted tumor tissues. $200 \mu \mathrm{l}$ of blank nanobubbles or targeted nanobubbles at the same concentration were injected into each nude mouse through the tail vein at random, and the tubing was washed with $100 \mu \mathrm{l}$ of normal saline. The images were continuously and dynamically collected. After $1 \mathrm{~h}$, nanobubbles in nude mice bodies completely disappeared, the same method was used to inject $200 \mu \mathrm{l}$ of nanobubbles suspension containing the same concentration of the other nanobubbles, which guaranteed nanobubbles would not interfere with the imaging effect of each other. The same process was used to collect ultrasound images of targeted and blank nanobubbles in liver and kidney tissues. The collected images were analyzed using Qlab8.1 software (Philips). Three indicators (peak time, peak intensity, and duration time) were compared for targeted and blank nanobubbles in liver and kidney,
CAIX-positive and CAIX-negative transplanted tumor tissues. Peak time referred to the time interval between the injection of nanobubbles and the time at which peak intensity was reached. Duration time referred to the time interval between the injection of nanobubbles and the completion of enhanced imaging.

\section{Immunohistochemistry identification}

The tumor-bearing nude mice were divided into two groups. After the completion of ultrasound contrast imaging, the nude mice in one group were injected with $200 \mu \mathrm{l}$ of DiI-labeled targeted nanobubbles through the tail vein. When peak intensity was achieved, the transplanted tumor tissues and the right thigh muscle tissues were collected and stored at $-20{ }^{\circ} \mathrm{C}$. The frozen specimens were placed on tissue holder, then added frozen embedding medium to solidify the specimens. After fixed in $4 \%$ paraformaldehyde, the sections were incubated with rat anti-mouse CD31 monoclonal antibody (Abcam, Cambridge, MA, USA) (1:300 dilution) at $4{ }^{\circ} \mathrm{C}$ overnight. FITC-labeled rabbit anti-rat secondary antibody (Beyotime Institute of Biotechnology) was added, and the sections were incubated for $2 \mathrm{~h}$ at room temperature in the dark. After washing with PBS, the sections were counterstained with DAPI for $10 \mathrm{~min}$, and observed under a LSCM. To further clarify the targeting ability of targeted nanobubbles, the expression of CD31 and CAIX were detected by immunohistochemical staining in paraffin sections. After the completion of ultrasound contrast imaging, the other mice were sacrificed and the transplanted tumor tissues were collected. Then the tumor tissues were fixed in paraformaldehyde, embedded in paraffin and sectioned. After routine deparaffination and blocking in $3 \% \mathrm{H}_{2} \mathrm{O}_{2}$, the sections were incubated with rabbit anti-mouse CD31 polyclonal antibody (Abcam) (1:100 dilution) or rabbit anti-human CAIX monoclonal antibody (Abcam) (1:400 dilution) at $4{ }^{\circ} \mathrm{C}$ overnight. Next, horseradish peroxidase-labeled goat anti-rabbit secondary antibody (Beyotime Institute of Biotechnology) was added, and the sections were incubated at $37^{\circ} \mathrm{C}$ for $1 \mathrm{~h}$. After in the presence of DAB reagent and counterstaining cell nuclei with hematoxylin, the sections were mounted and examined under an optical microscope. Microvessel density (MVD) was determined from five random fields (at $\times 200$ magnification) of each section, and only tumor blood vessels with vascular lumen or linear vascular shape were used for MVD analysis [25].

\section{Statistical analysis}

The Statistical Package for Social Sciences 22.0 (IBM Corporation, Armonk, NY, USA) was used for data analysis. All quantitative data are expressed as the mean \pm standard deviation. All parameter indicators were examined 
using paired $\mathrm{t}$ test and one-way analysis of variance. $\mathrm{P}<0.05$ indicated statistical significance. Histograms were plotted using GraphPad Prism 6.0 (GraphPad Software, Inc., La Jolla, CA, USA).

\section{Results \\ Synthesis and purification of polypeptides and FITC-modified polypeptides}

The polypeptides and the FITC-modified polypeptides were analyzed by high performance liquid chromatography, and both their purity was greater than $95 \%$. The relative molecular weight of the biotinylated polypeptide detected by mass spectrometry was $1722.2 \pm 0.4$, the theoretically calculated molecular weight of the biotinylated polypeptide was 1721.1 [see Additional file 1a]. The relative molecular weight of the FITC-modified biotinylated polypeptides was $2223.8 \pm 0.6$, the theoretically calculated molecular weight of the FITC-modified polypeptides was 2223.6 [see Additional file 1b]. Therefore, the relative molecular weight of the synthesis polypeptides and the synthesis FITC-modified polypeptides were consistent with the theoretically calculated molecular weight.

\section{Construction and identification of targeted nanobubbles}

The concentrations of targeted and blank nanobubbles were $(10.70 \pm 0.82) \times 10^{8} / \mathrm{ml}$ and $(11.53 \pm 0.81) \times 10^{8} /$ $\mathrm{ml}$, which were not significantly different $(\mathrm{P}>0.05)$. The particle sizes of targeted nanobubbles and blank nanobubbles determined with a Malvern Zetasizer nano ZS90 analyzer were $503.7 \pm 78.5 \mathrm{~nm}$ and $428.1 \pm 41.8 \mathrm{~nm}$, respectively (Fig. 1a). The zeta potential of blank nanobubbles was $-19.27 \pm 2.21 \mathrm{mV}$. The negative zeta potential of blank nanobubbles may be mainly ascribed to 1,2-dipalmitoyl-sn-glycero-3-phosphatidic acid, which is an anionic phospholipid [26]. When targeted nanobubbles were loaded with CAIX polypeptides, the zeta potential increased to $-14.80 \pm 2.12 \mathrm{mV}$, implying that the positively charged amino groups on CAIX polypeptides neutralized partial negative charge of anionic phospholipid [27]. Negative charge could keep the stability of targeted nanobubbles through the electrostatic repulsion. Under an optical microscope, targeted nanobubbles appeared evenly distributed, had similar particle sizes, did not display obvious aggregation or rupture, and showed good stability (Fig. 1b). Viewed by a transmission electron microscopy, they appeared regular and round in shape, with smooth boundaries, and the particle size was approximately $500 \mathrm{~nm}$ (Fig. 1c), which was consistent with the measurement results obtained with a Malvern Zetasizer nano ZS90 analyzer. The cytotoxicity of targeted and blank nanobubbles on 786-O cells was measured by MTT assay, which showed that the cytotoxicity of targeted nanobubbles and blank nanobubbles at the same concentrations was not significantly different $(\mathrm{P}>0.05)$, indicating that bound polypeptides did not significantly alter the cytotoxicity of nanobubbles (Fig. 1d). When the concentration of targeted nanobubbles at $2 \times 10^{7} / \mathrm{ml}$, the survival rate of the cells $(77.76 \% \pm 1.87 \%)$ was significantly lower than that $(90.23 \% \pm 1.52 \%)$ observed at the concentration of $2 \times 10^{6} / \mathrm{ml}(\mathrm{P}<0.05)$. The survival rate of 786-O cells was also different when the concentrations of blank nanobubbles were $2 \times 10^{7}$ and $2 \times 10^{6} / \mathrm{ml}$ $(\mathrm{P}<0.05)$.

Under a LSCM, lipid membrane of DiI-labeled targeted nanobubbles displayed red fluorescence (Fig. 2a), whereas the surfaces of targeted nanobubbles carrying the FITC-modified polypeptides displayed green fluorescence (Fig. 2b). These two types of fluorescence could overlap completely (Fig. 2c). Blank nanobubbles that did not carry the polypeptides and targeted nanobubbles that carried the unmodified polypeptides did not display green fluorescence.

The concentration of targeted nanobubbles decreased stored at $4{ }^{\circ} \mathrm{C}$, and were $(9.64 \pm 0.72) \times 10^{8}$, $(7.72 \pm 0.40) \times 10^{8},(4.40 \pm 0.45) \times 10^{8}$, $(3.11 \pm 0.44) \times 10^{8} / \mathrm{ml}$ at $1,2,4,7$ days, respectively (Fig. 3a). After 2 days, the concentration of targeted nanobubbles was different from that at 0 days $(\mathrm{P}<0.05)$. The particle size of targeted nanobubbles became larger over time, and were $567.2 \pm 80.4,690.8 \pm 95.5$, $889.6 \pm 99.7,1038 \pm 109.6 \mathrm{~nm}$ after stored for 1 , 2, 4, 7 days, respectively (Fig. 3b). After 4 days of storage, the particle size of targeted nanobubbles was different from that at 0 days $(\mathrm{P}<0.05)$.

\section{In vitro specific binding experiments with targeted nanobubbles}

The binding of targeted nanobubbles to the three types of cells was observed under an optical microscope (Fig. 4). The number of targeted nanobubbles binding to 786-O cells was significantly higher than that of blank nanobubbles binding to the cells, also higher than that of targeted nanobubbles binding to the cells pre-blocked by the polypeptides (Fig. $4 \mathrm{a}-\mathrm{C}$ ). The number of targeted nanobubbles binding to Hela cells was significantly higher than that of blank nanobubbles binding to the cells, also higher than that of targeted nanobubbles binding to the cells pre-blocked by the polypeptides (Fig. $4 \mathrm{~d}-\mathrm{f}$ ). The number of nanobubbles binding to BxPC-3 cells did not differ significantly in all groups (Fig. $4 \mathrm{~g}-\mathrm{i}$ ). Figure $4 \mathrm{j}$ showed the number of targeted nanobubbles and blank nanobubbles binding to the three types of tumor cells.

The affinity of targeted nanobubbles and blank nanobubbles to the three types of cells was determined by flow cytometry. The affinity of targeted nanobubbles to 786-O cells and HeLa cells was significantly higher than that of 

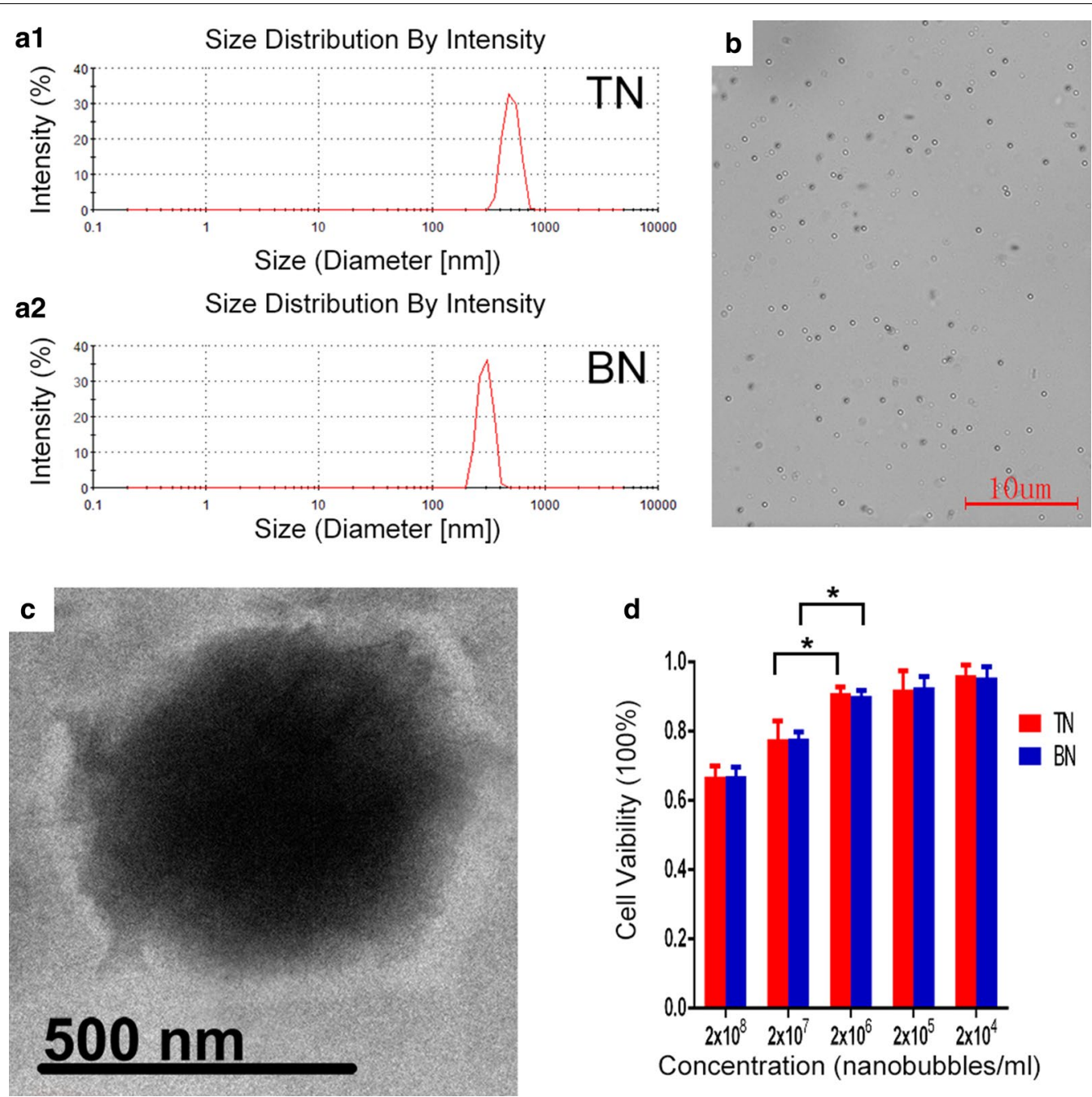

Fig. 1 Basic characteristics of targeted nanobubbles. a1 The particle size of targeted nanobubbles. a2 The particle size of blank nanobubbles. b The distribution of targeted nanobubbles under an optical microscope. c The morphology of targeted nanobubbles under a transmission electron microscope. $\mathbf{d}$ MTT assay of targeted and blank nanobubbles. ${ }^{*} \mathrm{P}<0.05$
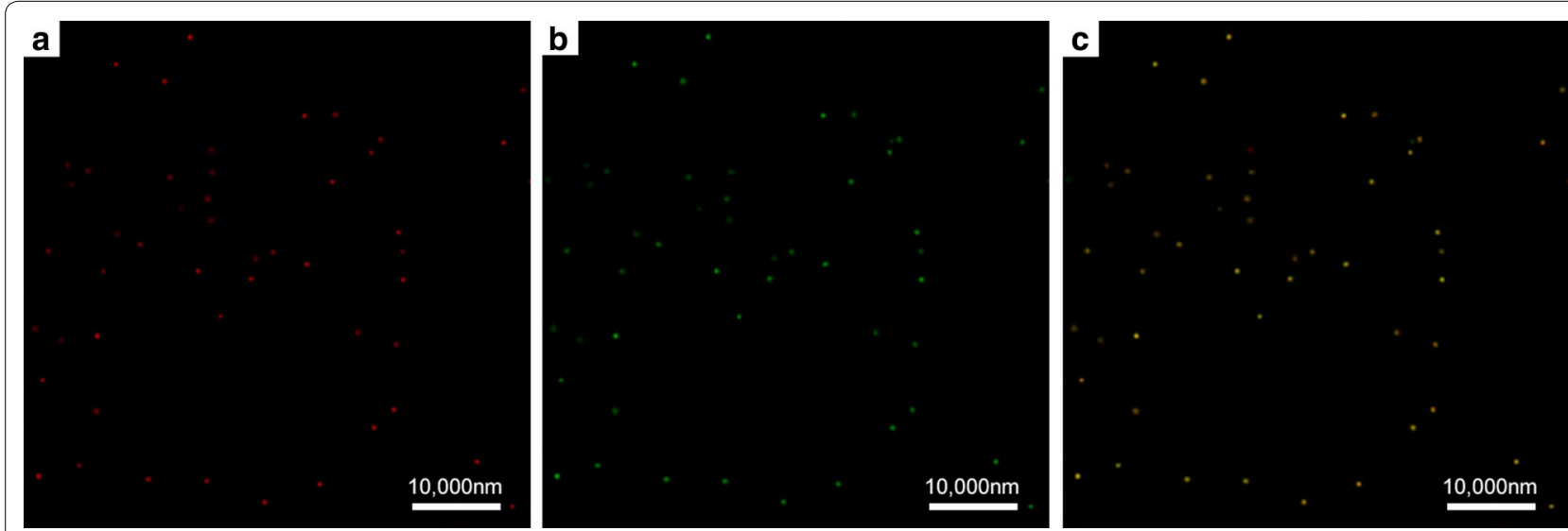

Fig. 2 Dual-fluorescence validates targeted nanobubbles. a Dil-labeled targeted nanobubbles. b Nanobubbles carrying the FITC-modified polypeptides. c Merged image confirms that biotin-streptavidin system successfully linked the polypeptides to the surfaces of targeted nanobubbles 

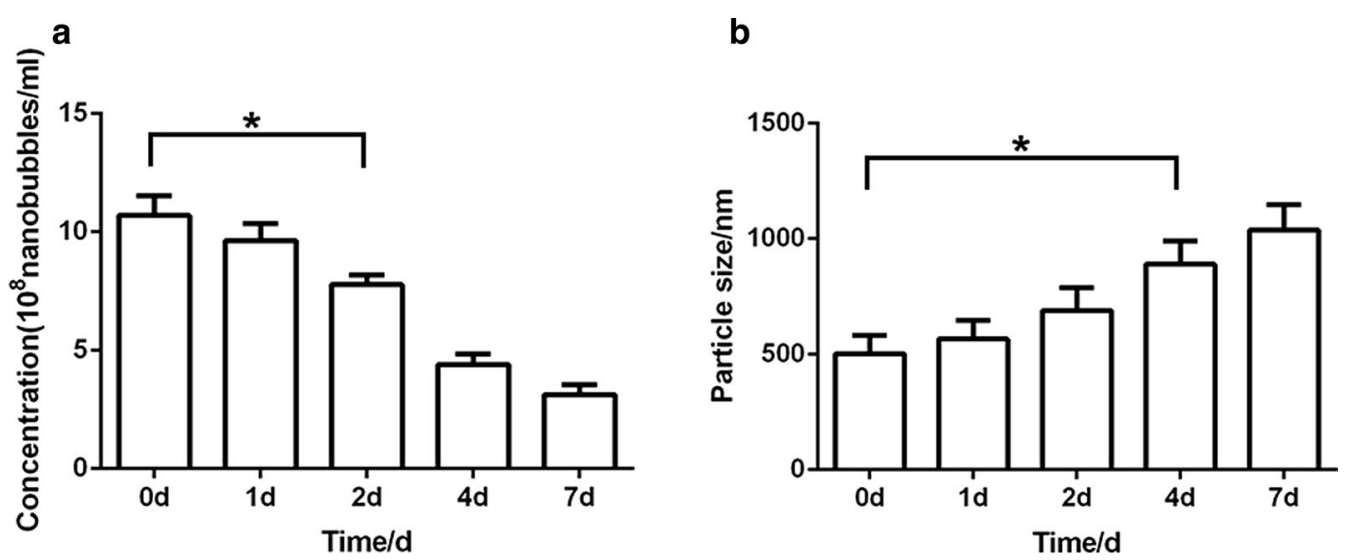

Fig. 3 Stability of targeted nanobubbles. a Histogram of the concentration of targeted nanobubbles changes over time. $\mathbf{b}$ Histogram of the particle size of targeted nanobubbles changes over time. ${ }^{*} \mathrm{P}<0.05$

blank nanobubbles to the two types of cells (Fig. 5a, b), whereas the affinity of the two types of nanobubbles to BxPC-3 cells did not differ significantly (Fig. 5c). Quantification of the affinity of targeted nanobubbles and blank nanobubbles to the three types of cells was shown in Fig. 5d. These results were consistent with the results obtained with an optical microscopy.

\section{In vitro imaging experiments with targeted nanobubbles}

In in vitro imaging model, the imaging intensity of targeted and blank nanobubbles at various concentration gradients did not show a significant difference (Fig. 6a). During 20 min of continuous and dynamic imaging in in vitro model, the imaging intensity of targeted and blank nanobubbles decreased over time. Linear fitting of the imaging intensity at different time points, taking the slope of the line as the decay rate of nanobubbles, the decay rate of targeted nanobubbles and blank nanobubbles was not significantly different $[28](\mathrm{P}>0.05)$ (Fig. 6b). After the "manual flash" technique was used, the imaging intensity of targeted nanobubbles was significantly decreased (Fig. 6c), indicating that targeted nanobubbles could be destructed by ultrasound waves with the high mechanical index.

\section{In vivo imaging experiments with targeted nanobubbles}

Targeted and blank nanobubbles were used to image liver, kidney and the three types of transplanted tumor tissues in nude mice. Peak time, peak intensity, and duration time of targeted nanobubbles and blank nanobubbles had no significant difference in liver, kidney, and BxPC-3 transplanted tumor tissues $(\mathrm{P}>0.05)$ (Table 1). In 786-O and HeLa transplanted tumor tissues, peak time of the two types of nanobubbles also had no significant difference $(P>0.05)$. However, peak intensity of targeted nanobubbles was significantly higher than that of blank nanobubbles $(\mathrm{P}<0.05)$. In addition, duration time of targeted nanobubbles was also significantly longer than that of blank nanobubbles $(\mathrm{P}<0.05)$ (Table 1 and Fig. $7 \mathrm{a}$ ). Peak intensity and duration time of targeted nanobubbles in 786-O and HeLa transplanted tumor tissues were also significantly superior to those in BxPC-3 transplanted tumor tissues $(\mathrm{P}<0.05)$ (Table 1 and Fig. 7a). Dynamic analysis of the time-intensity curves of ultrasound imaging in the three types of transplanted tumor tissues from 0 to $15 \mathrm{~min}$, the imaging intensity of targeted nanobubbles was always higher than that of blank nanobubbles in $786-\mathrm{O}$ and HeLa transplanted tumor tissues $(\mathrm{P}<0.05)$, while the imaging intensity of targeted nanobubbles was not different from blank nanobubbles in BxPC-3 transplanted tumor tissues $(\mathrm{P}>0.05)$ (Fig. 7b). Moreover, in 786-O and Hela transplanted tumor tissues, the difference of imaging intensity between targeted nanobubbles and blank nanobubbles increased over time from $0 \mathrm{~min}$ to $5 \mathrm{~min}$, while the difference decreased over time from 5 min to 15 min. In 786-O and HeLa transplanted tumor tissues, area under the curve of targeted nanobubbles was significantly larger than that of blank nanobubbles (11792.49 \pm 453.32 vs $8498.74 \pm 449.75$ and $11658.93 \pm 431.65$ vs $8348.65 \pm 289.44$ ), while area under the curve of targeted nanobubbles was not significantly different from blank nanobubbles in BxPC-3 transplanted tumor tissues $(8262.62 \pm 267.28$ vs $8328.89 \pm 209.33)$ (Fig. 7c).

\section{Localization of targeted nanobubbles in transplanted tumor tissues}

Observation of the frozen sections of the transplanted tumor tissues and the right thigh muscle tissues after injection of DiI-labeled targeted nanobubbles, targeted 


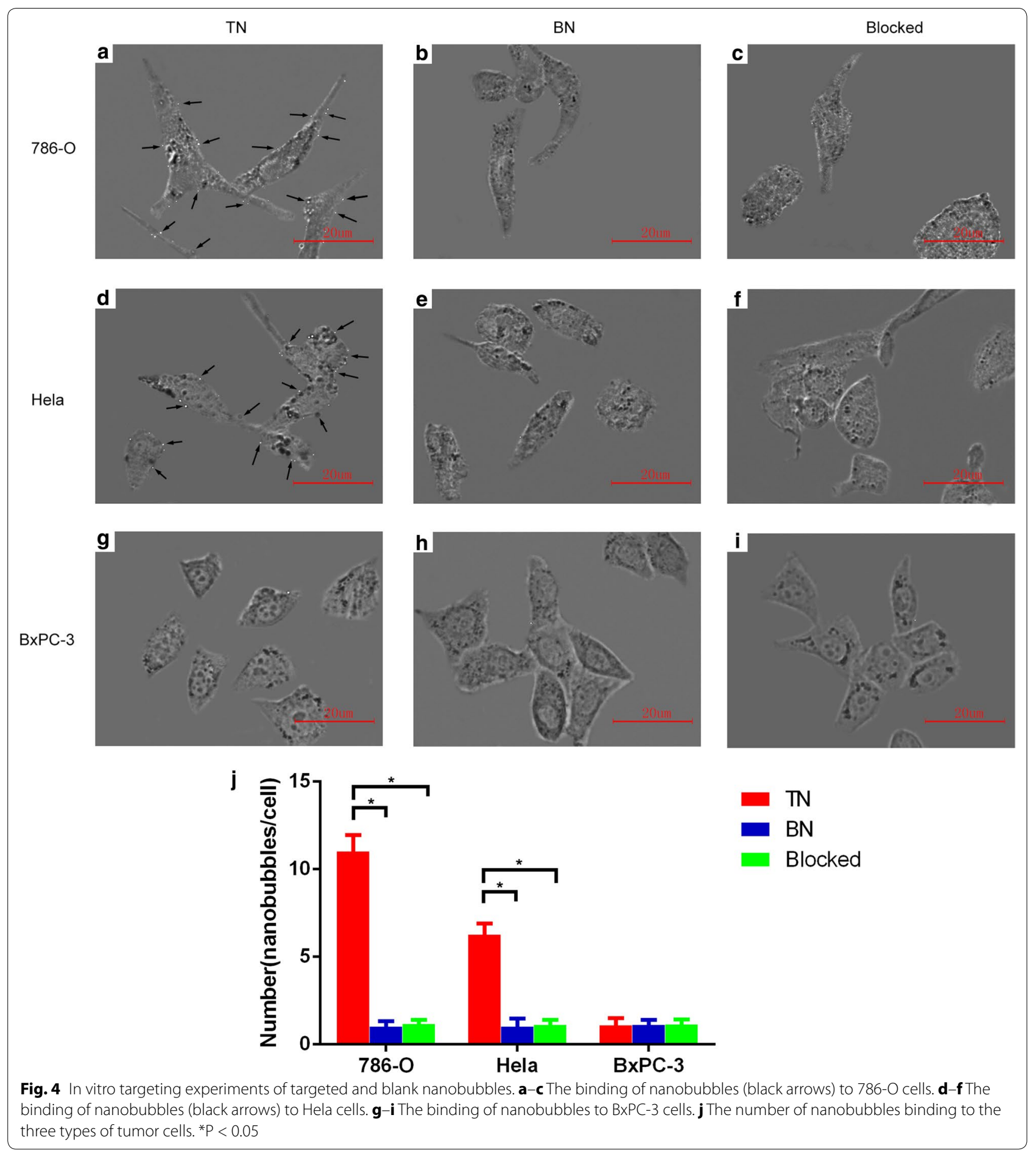

nanobubbles were distributed in tumor blood vessels and tissue spaces outside blood vessels in 786-O, Hela, and BxPC-3 transplanted tumor tissues (Fig. 8a-l). Targeted nanobubbles with intact morphology could occasionally be observed in tissue spaces. While targeted nanobubbles were only present in blood vessels, and no targeted nanobubbles in tissue spaces in the right thigh muscle tissues (Fig. $8 \mathrm{~m}-$ p). Targeted nanobubbles in 786-O and HeLa transplanted tumor tissue spaces were significantly more than those in BxPC-3 transplanted tumor tissue spaces. 

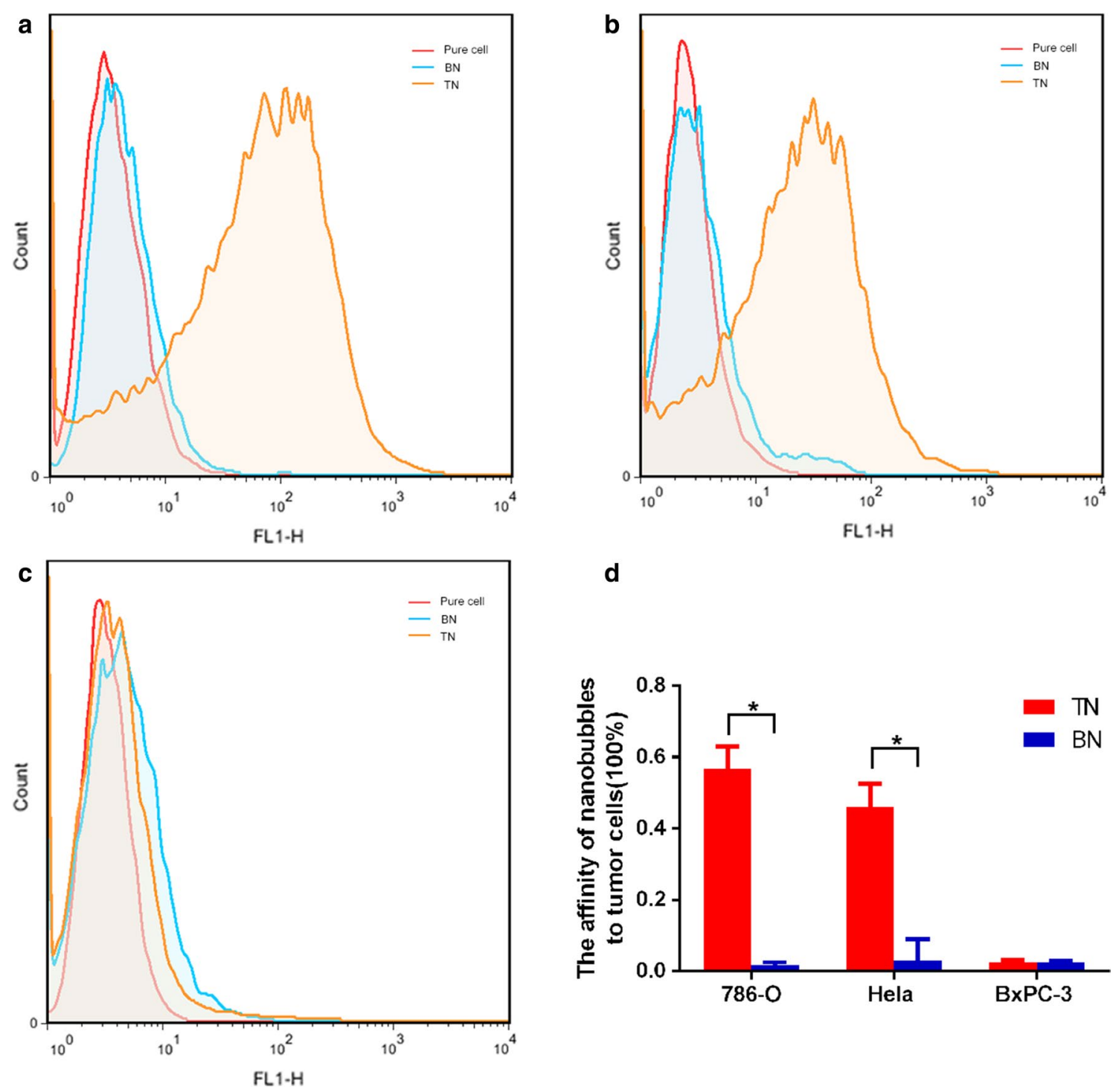

d

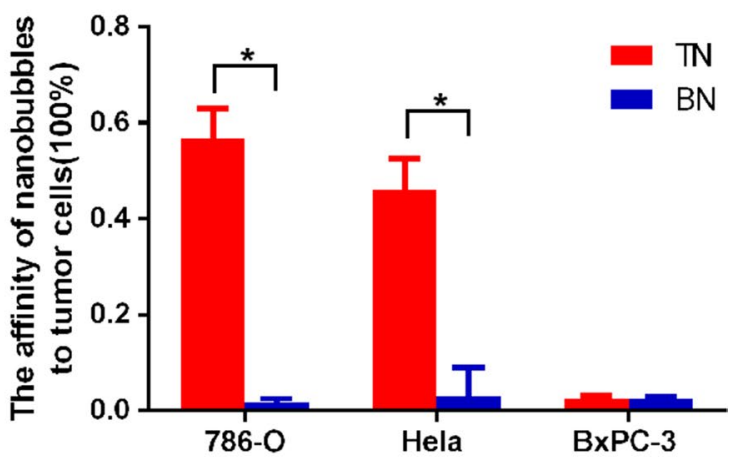

Fig. 5 Affinity of targeted and blank nanobubbles to three types of cells. a The affinity of nanobubbles to 786-O cells. b The affinity of nanobubbles to HeLa cells. c The affinity of nanobubbles to BxPC-3 cells. d Quantification of the affinity of the two types of nanobubbles to the three types of cells. ${ }^{*} \mathrm{P}<0.05$

\section{MVD and CAIX expression in transplanted tumor tissues}

To clarify the reason of the difference in distribution of targeted nanobubbles in the three types of transplanted tumor tissues, we analyzed MVD and CAIX expression in them. Blood vessels that stained dark brown were mainly distributed in tumor stroma that infiltrated into tumor parenchyma in 786-O transplanted tumor tissues and MVD was $19.20 \pm 2.68$ (Fig. 9a). Blood vessels that stained dark brown were distributed in tumor stroma that wrapped around tumor parenchyma in HeLa and BxPC-3 transplanted tumor tissues and MVDs were $20.20 \pm 2.28$ and $17.40 \pm 1.67$, respectively (Fig. 9b, c).
While MVDs in the three types of transplanted tumor tissues were not different (Fig. 9d). In 786-O and Hela transplanted tumor tissues, only tumor parenchyma cells (yellow-brown areas) expressed CAIX, and tumor stroma cells (black arrows) and necrotic areas did not express CAIX (Fig. 10a, b), while no BxPC-3 transplanted tumor tissues expressed CAIX (Fig. 10c). Some apparent vascular structures were visible in tumor stroma of the three types of transplanted tumor tissues, which did not express CAIX. 


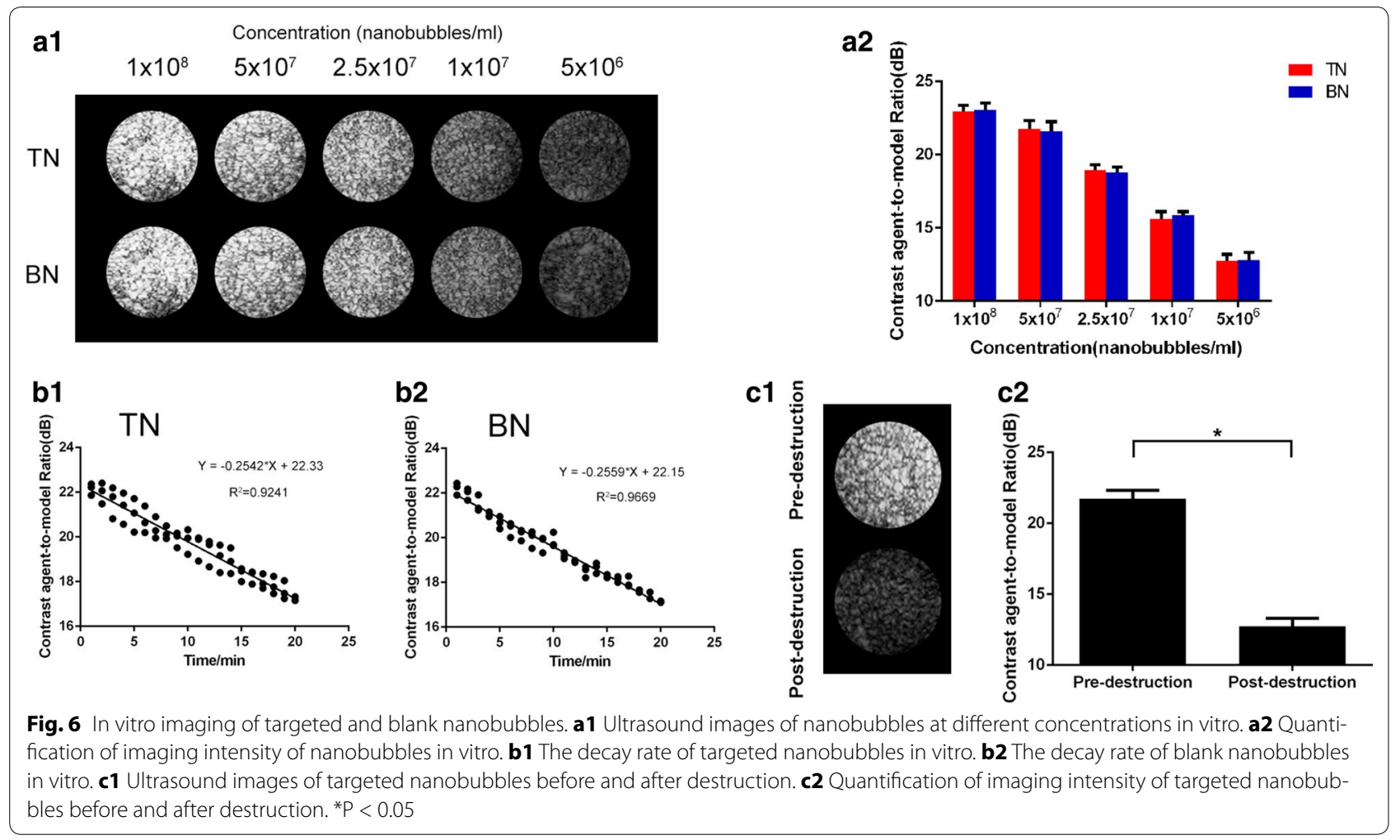

Table 1 Ultrasound parameters of targeted and blank nanobubbles in liver, kidney and transplanted tumors

\begin{tabular}{|c|c|c|c|c|}
\hline Tissue & Contrast agent & Peak time/s & Peak intensity/dB & Duration time/min \\
\hline \multirow[t]{2}{*}{ Liver } & $\mathrm{TN}$ & $6.22 \pm 0.50$ & $18.31 \pm 0.61$ & $21.46 \pm 0.87$ \\
\hline & $\mathrm{BN}$ & $6.32 \pm 0.95$ & $18.52 \pm 0.95$ & $21.19 \pm 1.68$ \\
\hline \multirow[t]{2}{*}{ Kidney } & TN & $2.01 \pm 0.34$ & $17.48 \pm 0.76$ & $20.11 \pm 1.20$ \\
\hline & BN & $2.37 \pm 0.59$ & $17.42 \pm 0.87$ & $19.77 \pm 0.77$ \\
\hline \multirow[t]{2}{*}{ 786-O tumor } & $\mathrm{TN}$ & $8.28 \pm 0.74$ & $21.56 \pm 0.62^{*, \#}$ & $21.35 \pm 0.51^{*, \#}$ \\
\hline & BN & $8.44 \pm 0.71$ & $19.30 \pm 0.68$ & $17.91 \pm 0.81$ \\
\hline \multirow[t]{2}{*}{ Hela tumor } & $\mathrm{TN}$ & $8.31 \pm 0.75$ & $20.51 \pm 0.69^{*, \#}$ & $20.93 \pm 0.61^{* \#}$ \\
\hline & $\mathrm{BN}$ & $8.25 \pm 0.78$ & $18.53 \pm 0.42$ & $17.99 \pm 0.85$ \\
\hline \multirow[t]{2}{*}{ BxPC-3 tumor } & TN & $8.04 \pm 0.85$ & $18.34 \pm 0.65$ & $17.55 \pm 0.53$ \\
\hline & $\mathrm{BN}$ & $8.41 \pm 0.43$ & $18.53 \pm 0.60$ & $17.66 \pm 0.84$ \\
\hline
\end{tabular}

* Compared with the same indicator from blank nanobubbles, $\mathrm{P}<0.05$

\# Compared with the same indicator from BxPC-3 transplanted tumor tissues, $\mathrm{P}<0.05$

\section{Discussion}

Malignant tumors severely affect human life and health. Early diagnosis and timely treatment contribute greatly to increasing the quality of life and improving the prognosis of cancer patients, especially the common malignant tumors such as cervical cancer and kidney cancer $[29,30]$. Due to conventional ultrasound imaging techniques do not reveal the change of molecular expression in malignant tumors prior to the change in anatomical structures, early diagnosis and timely treatment of malignant tumors cannot be truly realized [31]. Through tumor-targeted ultrasound molecular probes (i.e., targeted UCAs), ultrasound molecular imaging provides a novel method for early identification of the change in molecular expression in malignant tumors.

UCAs currently used for molecular imaging of tumors have two main disadvantages. (1) UCAs are mostly at micron scale, so they can only achieve imaging in blood pool and cannot aggregate in tumor tissues to achieve specific imaging of tumor parenchymal cells [32-35]. For 


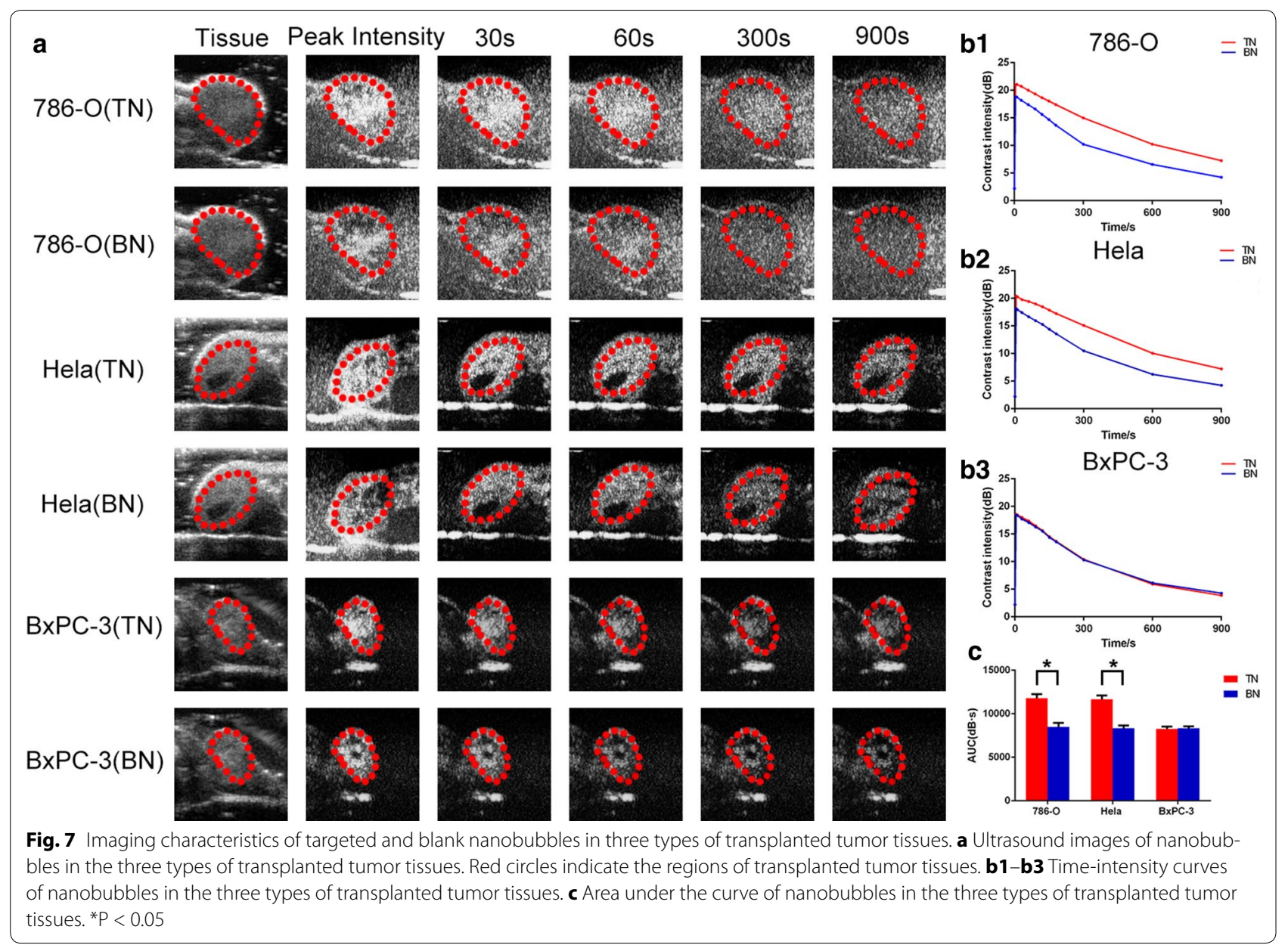

example, BR55 that targets vascular endothelial growth factor receptor (VEGFR) has been used in tumor diagnosis [4]. The EPR effect of tumor blood vessels theoretically only allows nanoscale UCAs (e.g., nanobubbles) pass through tumor blood vessels and achieve targeted ultrasound molecular imaging of stroma and parenchymal cells of tumor tissues [36-39]. (2) Targeted UCAs generally aim at only one type of tumor, and only can be used in early diagnosis of one particular malignant tumor, so their clinical application value is limited. For example, we were only able to perform targeted ultrasound imaging of prostate cancer and metastasis and could not perform targeted ultrasound imaging of other tumors in our previous studies [13].

To construct targeted UCAs that specifically aggregate in tumor tissues and achieve ultrasound molecular imaging of a variety of tumors, it is necessary to link ligands that have common specific targets in a variety of tumors to UCAs [40]. Previous studies showed that CAIX is highly expressed on cell membranes of a variety of malignant tumors, including lung, kidney, colon and rectum, breast, cervix, head and neck, and bladder cancer. Due to extracellular proteoglycan domain of CAIX is a specific structure, ligands that target proteoglycan domain of CAIX do not cross-react with other carbonic anhydrase isoenzymes [14].

Polypeptide molecules are ideal ligands in molecular imaging, because their relatively low molecular weights, high selectivity and specificity, strong cell permeability, easily modified without affecting specific characteristics, and low immunogenicity [18, 41, 42]. PGLR-P1 polypeptides specifically bind to CAIX protein, and smaller than other ligand, such as aptamer and monoclonal antibodies. Moreover, there are no current studies on the construction of targeted nanobubbles that carry polypeptides targeting parenchymal cells or targeted ultrasound molecular imaging of many tumors.

Combining the advantages of CAIX, polypeptides and nanobubbles, we used biotin-streptavidin system to construct targeted nanobubbles carrying CAIX polypeptides in the study, whose particle size was only $503.7 \pm 78.47 \mathrm{~nm}$. The novel targeted nanobubbles 


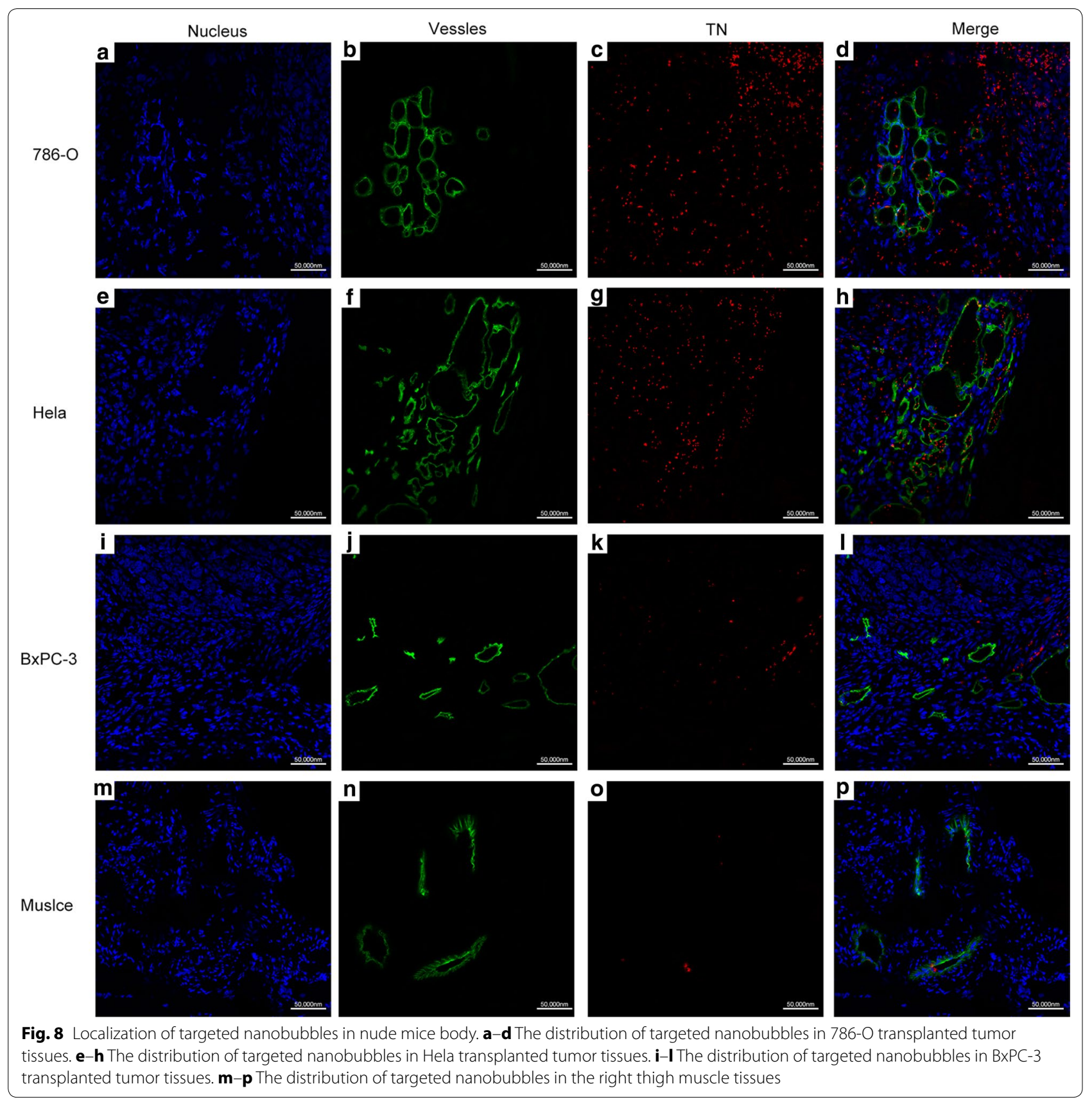

possessed the advantages of homogenous particle size, high stability, and good safety. We used DiI to label lipid membranes of targeted nanobubbles and FITC to modify the polypeptides. Under a LSCM, green fluorescence and red fluorescence could completely overlap, indicating targeted nanobubbles were constructed successfully and the polypeptides evenly distributed on the surfaces of targeted nanobubbles. MTT assay showed that the cytotoxicity of targeted nanobubbles and blank nanobubbles was not significantly different. Targeted nanobubbles carrying
CAIX polypeptides specifically aggregated on CAIX-positive tumor cells in vitro at the cellular level.

To analyze ultrasound imaging characteristics of targeted UCAs in vivo is extremely important in ultrasound molecular imaging. Peak time, peak intensity, and duration time of targeted and blank nanobubbles in CAIXnegative tissues (liver, kidney and BxPC-3 transplanted tumor tissues) were not different. While peak intensity and duration time of targeted nanobubbles were significantly better than those of blank nanobubbles in 

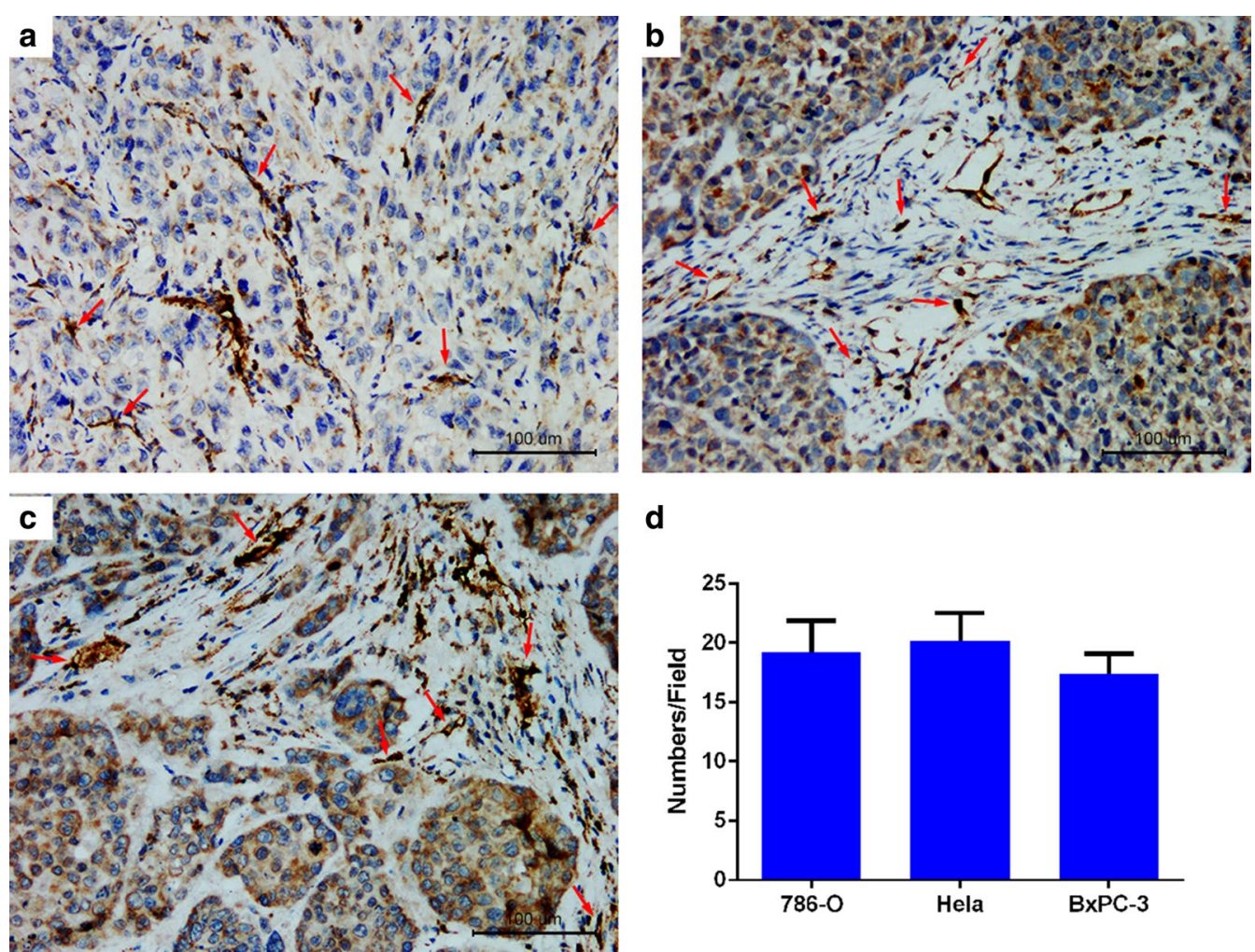

d

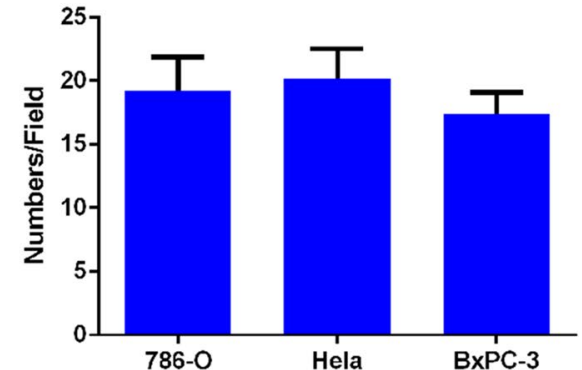

Fig. 9 Blood vessels in three types of transplanted tumor tissues. a Blood vessels in 786-O transplanted tumor tissues. b Blood vessels in Hela transplanted tumor tissues. c Blood vessels in BxPC-3 transplanted tumor tissues. d MVDs in the three types of transplanted tumor tissues were assessed. Red arrows indicate tumor blood vessels
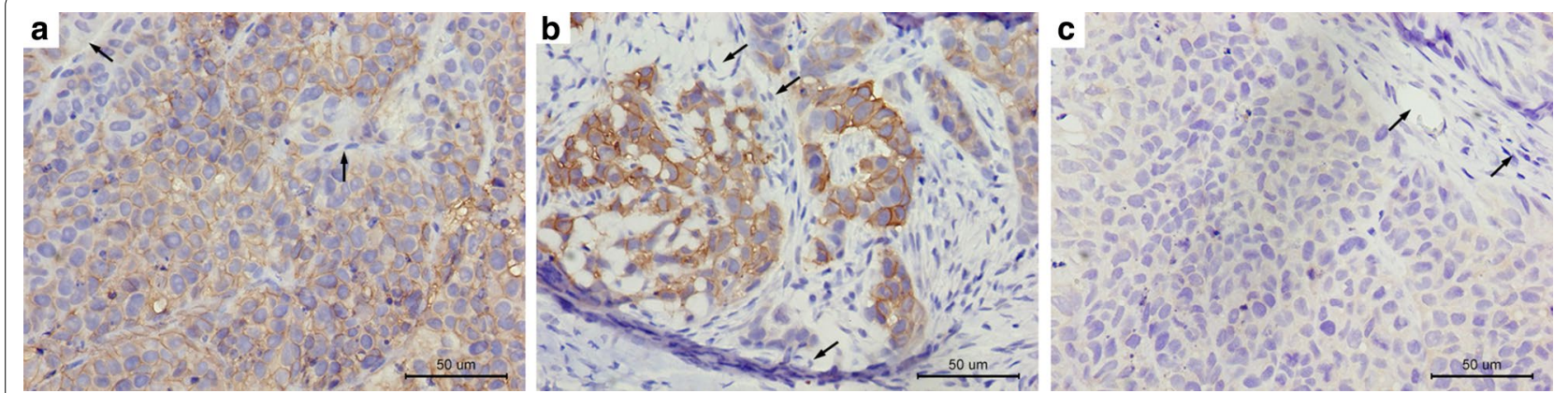

Fig. 10 Expression of CAIX in three types of transplanted tumor tissues. a Immunohistochemistry on 786-O transplanted tumor tissues. $\mathbf{b}$ Immunohistochemistry on Hela transplanted tumor tissues. c Immunohistochemistry on BxPC-3 transplanted tumor tissues. The yellow-brown areas indicate positive CAIX expression, black arrows indicate tumor stroma

CAIX-positive tissues (786-O and Hela transplanted tumor tissues), and also significantly better than those of targeted nanobubbles in CAIX-negative tissues (BxPC-3 transplanted tumor tissues). The reason probably is that targeted nanobubbles carrying CAIX polypeptides actively aggregated in CAIX-positive tumor tissues, so the number of targeted nanobubbles in the cross-sections of CAIX-positive transplanted tumor tissues was significantly more than others, including the number of blank nanobubbles in CAIX-positive transplanted tumor tissues and the number of targeted nanobubbles in CAIXnegative transplanted tumor tissues. Combined with the feature of nanobubbles aggregation imaging, the imaging effect of targeted nanobubbles was better than that of 
blank nanobubbles in CAIX-positive transplanted tumor tissues, also better than that of targeted nanobubbles in CAIX-negative transplanted tumor tissues, which was consistent with the results of Cai et al. [9, 24, 43-45].

The basis of ultrasound molecular imaging is that targeted nanobubbles penetrate blood vessels and enter into transplanted tumors tissue spaces, so that to determine the distribution of targeted nanobubbles in different types of transplanted tumor tissues is important. We found targeted nanobubbles could pass through tumor blood vessels and reach the three types of transplanted tumor tissue spaces, while could not be distributed in the right thigh muscle tissue spaces. Meanwhile, we found that a number of targeted nanobubbles were distributed in CAIX-positive transplanted tumor tissues (786-O and HeLa transplanted tumor tissues) and little targeted nanobubbles were distributed in CAIX-negative transplanted tumor tissues (BxPC-3 transplanted tumor tissues).

Considering tumor blood vessels play an important role in CEUS imaging, we analyzed MVD of the transplanted tumor tissues [43]. However, MVDs were not significant different in the three types of transplanted tumor tissues, so tumor blood vessel was not the reason of the imaging differences of targeted nanobubbles in the three types of transplanted tumor tissues. At last, we confirmed 786-O and HeLa transplanted tumor tissues expressed CAIX, while BxPC-3 transplanted tumor tissues did not express CAIX. Therefore, the expression of CAIX resulted in the imaging difference of targeted nanobubbles in the three types of transplanted tumor tissues, which could promote targeted nanobubbles to aggregate in CAIX-positive transplanted tumor tissues and then enhanced transplanted tumor imaging.

Due to targeted nanobubbles carrying CAIX polypeptides could pass through tumor blood vessels and specifically enhanced ultrasound molecular imaging in CAIX-positive transplanted tumor tissues, ultrasound molecular imaging could find the change of CAIX expression prior to the change in anatomical structures and achieve early diagnosis of many solid tumors derived from various organs. Though some studies have confirmed that the binding ability and imaging effect of blank microbubbles without carrying ligand were nearly identical with those of scrambled microbubbles carrying nonsense ligand, and the two kinds of microbubbles had a poorer capacity than those of targeted microbubbles carrying specific ligand in positive expression tissues, scrambled nanobubbles carrying nonsense polypeptides as the negative control may be more complete $[46,47]$.

\section{Conclusions}

In summary, we used biotin-streptavidin system to construct targeted nanobubbles carrying CAIX polypeptides, which possessed the advantage of excellent penetration ability and strong specificity. In addition, dual-fluorescence confirmed the polypeptides evenly distributed on the surfaces of targeted nanobubbles and immunofluorescence confirmed targeted nanobubbles specifically aggregated in CAIX-positive tumor tissues. This study not only provides a novel ultrasound molecular probe for targeted ultrasound molecular imaging of a variety of tumors derived from various organs, but also further describes targeted nanobubbles could pass through tumor blood vessels to enter in tumor tissue spaces and clarifies the imaging differences of targeted nanobubbles in different types of transplanted tumor tissues.

\section{Additional file}

Additional file 1. Mass spectrogram of polypeptides. a Mass spectrogram of the unmodified polypeptides. b Mass spectrogram of the FITCmodified polypeptides.

\section{Abbreviations \\ CAIX: carbonic anhydrase IX; CE-MRI: contrast-enhanced magnetic resonance imaging; CE-CT: contrast-enhanced computed tomography; CEUS: contrast- enhanced ultrasound; UCAs: ultrasound contrast agents; EPR: enhanced permeability and retention; PBS: phosphate-buffered saline; TN: targeted nanobubbles; BN: blank nanobubbles; DMSO: dimethyl sulphoxide; BSA: bovine serum albumin; LSCM: laser scanning confocal microscope; MVD: microvessel density; VEGFR: vascular endothelial growth factor receptor.}

\section{Authors' contributions}

YLG, LFW and LHZ conceived and designed the experiments. LHZ, XZF, and $X Y X$ performed the experiments. LHZ, KJF and DX analyzed the data. LHZ and LFW prepared all the figures. LHZ, YLG and XZF prepared the manuscript. All authors read and approved the final manuscript.

\section{Author details}

${ }^{1}$ Department of Ultrasound, Southwest Hospital, Third Military Medical University, 30 Gaotanyan Street, Shapingba District, Chongqing 400038, China. ${ }^{2}$ Department of Urology, Daping Hospital, Third Military Medical University, 10 Changjiang Zhi Road, Yuzhong District, Chongqing 400038, China.

\section{Acknowledgements}

Not applicable.

\section{Competing interests}

The authors declare that they have no competing interests.

\section{Availability of data and materials}

All data generated or analyzed during this study are included in this published article and its Additional information files.

\section{Consent for publication}

Not applicable.

\section{Ethics approval and consent to participate}

The study was approved by the Laboratory Animal Welfare and Ethics Committee of the Third Military Medical University and was performed in accordance with the NIH Guide for the Care and Use of Laboratory Animals. 


\section{Funding}

This work was supported by International Science \& Technology Cooperation Program of China (No. 2015DFA30920), National Natural Science Foundation of China (No. 81571732) and Science and Technology (International Science \& Technology Cooperation) Research Base Construction Program of Chongqing (No. cstc2014gjhz110004). All funders had no role in study design, data collection and analysis, decision to publish, or preparation of the manuscript.

\section{Publisher's Note}

Springer Nature remains neutral with regard to jurisdictional claims in published maps and institutional affiliations.

\section{Received: 15 May 2017 Accepted: 23 September 2017} Published online: 29 September 2017

\section{References}

1. Pitre-Champagnat S, Leguerney I, Bosq J, Peronneau P, Kiessling F, Calmels L, Coulot J, Lassau N. Dynamic contrast-enhanced ultrasound parametric maps to evaluate intratumoral vascularization. Invest Radiol. 2015:50:212-7.

2. Luo W, Wen G, Yang L, Tang J, Wang J, Wang J, Zhang S, Zhang L, Ma F, Xiao L, et al. Dual-targeted and $\mathrm{pH}$-sensitive doxorubicin prodrugmicrobubble complex with ultrasound for tumor treatment. Theranostics. 2017:7:452-65.

3. Wang J, Qin B, Chen X, Wagner WR, Villanueva FS. Ultrasound molecular imaging of angiogenesis using vascular endothelial growth factor-conjugated microbubbles. Mol Pharm. 2017;14:781-90.

4. Abou-Elkacem L, Bachawal SV, Willmann JK. Ultrasound molecular imaging: moving toward clinical translation. Eur J Radiol. 2015:84:1685-93.

5. Xing Z, Wang J, Ke H, Zhao B, Yue X, Dai Z, Liu J. The fabrication of novel nanobubble ultrasound contrast agent for potential tumor imaging. Nanotechnology. 2010;21:145607

6. Unnikrishnan S, Klibanov AL. Microbubbles as ultrasound contrast agents for molecular imaging: preparation and application. AJR Am J Roentgenol. 2012;199:292-9.

7. Paefgen V, Doleschel D, Kiessling F. Evolution of contrast agents for ultrasound imaging and ultrasound-mediated drug delivery. Front Pharmacol. 2015:6:197.

8. Yin T, Wang P, Zheng R, Zheng B, Cheng D, Zhang X, Shuai X. Nanobubbles for enhanced ultrasound imaging of tumors. Int J Nanomed. 2012;7:895-904

9. Cai WB, Yang HL, Zhang J, Yin JK, Yang YL, Yuan LJ, Zhang L, Duan YY. The optimized fabrication of nanobubbles as ultrasound contrast agents for tumor imaging. Sci Rep. 2015:5:13725.

10. Miller AD. Lipid-based nanoparticles in cancer diagnosis and therapy. J Drug Deliv. 2013;2013:165981.

11. Fan X, Wang L, Guo Y, Tu Z, Li L, Tong H, Xu Y, Li R, Fang K. Ultrasonic nanobubbles carrying Anti-PSMA nanobody: construction and application in prostate cancer-targeted imaging. PLOS ONE. 2015;10:e0127419.

12. Wang L, Li L, Guo Y, Tong H, Fan X, Ding J, Huang H. Construction and in vitro/in vivo targeting of PSMA-targeted nanoscale microbubbles in prostate cancer. Prostate. 2013;73:1147-58.

13. Fan $X$, Guo $Y$, Wang $L$, Xiong $X$, Zhu L, Fang $K$. Diagnosis of prostate cancer using anti-PSMA aptamer A10-3.2-oriented lipid nanobubbles. Int J Nanomed. 2016:11:3939-50.

14. Mahon BP, Pinard MA, McKenna R. Targeting carbonic anhydrase IX activity and expression. Molecules. 2015:20:2323-48.

15. Tafreshi NK, Lloyd MC, Bui MM, Gillies RJ, Morse DL. Carbonic anhydrase IX as an imaging and therapeutic target for tumors and metastases. Subcell Biochem. 2014;75:221-54.

16. Pastorek J, Pastorekova S. Hypoxia-induced carbonic anhydrase IX as a target for cancer therapy: from biology to clinical use. Semin Cancer Biol. 2015;31:52-64.

17. $\mathrm{Ng} \mathrm{HL}$, Lu A, Lin G, Qin L, Yang Z. The potential of liposomes with carbonic anhydrase IX to deliver anticancer ingredients to cancer cells in vivo. Int J Mol Sci. 2014:16:230-55.
18. Liu J, Liu J, Xu H, Zhang Y, Chu L, Liu Q, Song N, Yang C. Novel tumortargeting, self-assembling peptide nanofiber as a carrier for effective curcumin delivery. Int J Nanomed. 2014;9:197-207.

19. Fokong S, Fragoso A, Rix A, Curaj A, Wu Z, Lederle W, Iranzo O, Gatjens J, Kiessling F, Palmowski M. Ultrasound molecular imaging of E-selectin in tumor vessels using poly n-butyl cyanoacrylate microbubbles covalently coupled to a short targeting peptide. Invest Radiol. 2013:48:843-50.

20. van Rooij T, Daeichin V, Skachkov I, de Jong N, Kooiman K. Targeted ultrasound contrast agents for ultrasound molecular imaging and therapy. Int J Hyperth. 2015;31:90-106.

21. Chen ZY, Wang YX, Lin Y, Zhang JS, Yang F, Zhou QL, Liao YY. Advance of molecular imaging technology and targeted imaging agent in imaging and therapy. Biomed Res Int. 2014;2014:819324.

22. Rana S, Nissen F, Lindner T, Altmann A, Mier W, Debus J, Haberkorn U, Askoxylakis V. Screening of a novel peptide targeting the proteoglycanlike region of human carbonic anhydrase IX. Mol Imaging. 2013;12:7290.

23. Negishi Y, Hamano N, Tsunoda Y, Oda Y, Choijamts B, Endo-Takahashi Y, Omata D, Suzuki R, Maruyama K, Nomizu M, et al. AG73-modified bubble liposomes for targeted ultrasound imaging of tumor neovasculature. Biomaterials. 2013:34:501-7.

24. Yang H, Cai W, Xu L, Lv X, Qiao Y, Li P, Wu H, Yang Y, Zhang L, Duan Y. Nanobubble-Affibody: novel ultrasound contrast agents for targeted molecular ultrasound imaging of tumor. Biomaterials. 2015;37:279-88.

25. Zhu $Q$, Jiang $L$, Wang $X$. The expression of duffy antigen receptor for chemokines by epithelial ovarian cancer decreases growth potential. Oncol Lett. 2017:13:4302-6.

26. Lim JL, Ki MH, Joo MK, An SW, Hwang KM, Park ES. An injectable liquid crystal system for sustained delivery of entecavir. Int J Pharm. 2015:490:265-72

27. Cheng W, Nie J, Xu L, Liang C, Peng Y, Liu G, Wang T, Mei L. pH-Sensitive delivery vehicle based on folic acid-conjugated polydopamine-modified mesoporous silica nanoparticles for targeted cancer therapy. ACS Appl Mater Interfaces. 2017:9:18462-73.

28. Wu H, Rognin NG, Krupka TM, Solorio L, Yoshiara H, Guenette G, Sanders C, Kamiyama N, Exner AA. Acoustic characterization and pharmacokinetic analyses of new nanobubble ultrasound contrast agents. Ultrasound Med Biol. 2013;39:2137-46.

29. Chen W, Zheng $\mathrm{R}$, Zeng $\mathrm{H}$, Zhang $\mathrm{S}$, He J. Annual report on status of cancer in China, 2011. Chin J Cancer Res. 2015;27:2-12

30. Chen W, Zheng R, Baade PD, Zhang S, Zeng H, Bray F, Jemal A, Yu XQ, He J. Cancer statistics in China, 2015. CA Cancer J Clin. 2016;66:115-32.

31. Thakor AS, Gambhir SS. Nanooncology: the future of cancer diagnosis and therapy. CA Cancer J Clin. 2013;63:395-418.

32. Bettinger T, Bussat P, Tardy I, Pochon S, Hyvelin JM, Emmel P, Henrioud S, Biolluz N, Willmann JK, Schneider M, Tranquart F. Ultrasound molecular imaging contrast agent binding to both $\mathrm{E}$ - and P-selectin in different species. Invest Radiol. 2012;47:516-23.

33. Wang H, Kaneko OF, Tian L, Hristov D, Willmann JK. Three-dimensional ultrasound molecular imaging of angiogenesis in colon cancer using a clinical matrix array ultrasound transducer. Invest Radiol. 2015;50:322-9.

34. Hu Q, Wang XY, Kang LK, Wei HM, Xu CM, Wang T, Wen ZH. RGD-Targeted ultrasound contrast agent for longitudinal assessment of Hep-2 tumor angiogenesis in vivo. PLoS ONE. 2016;11:e0149075.

35. Varga A, Gyulavari P, Greff Z, Futosi K, Nemeth T, Simon-Szabo L, Kerekes K, Szantai-Kis C, Brauswetter D, Kokas M, et al. Targeting vascular endothelial growth factor receptor 2 and protein kinase D1 related pathways by a multiple kinase inhibitor in angiogenesis and inflammation related processes in vitro. PLoS ONE. 2015;10:e0124234.

36. Jiang Q, Hao S, Xiao X, Yao J, Ou B, Zhao Z, Liu F, Pan X, Luo B, Zhi H. Production and characterization of a novel long-acting herceptin-targeted nanobubble contrast agent specific for Her-2-positive breast cancers. Breast Cancer. 2016:23:445-55.

37. Tong HP, Wang LF, Guo YL, Li L, Fan XZ, Ding J, Huang HY. Preparation of protamine cationic nanobubbles and experimental study of their physical properties and in vivo contrast enhancement. Ultrasound Med Biol. 2013:39:2147-57.

38. Marxer EE, Brussler J, Becker A, Schummelfeder J, Schubert R, Nimsky C, Bakowsky U. Development and characterization of new nanoscaled ultrasound active lipid dispersions as contrast agents. Eur J Pharm Biopharm. 2011:77:430-7. 
39. Fan $X$, Wang L, Guo Y, Tong H, Li L, Ding J, Huang H. Experimental investigation of the penetration of ultrasound nanobubbles in a gastric cancer xenograft. Nanotechnology. 2013;24:325102.

40. Kwon IK, Lee SC, Han B, Park K. Analysis on the current status of targeted drug delivery to tumors. J Control Release. 2012;164:108-14.

41. Ackerman SE, Currier NV, Bergen JM, Cochran JR. Cystine-knot peptides: emerging tools for cancer imaging and therapy. Expert Rev Proteom. 2014;11:561-72.

42. Omidfar K, Daneshpour M. Advances in phage display technology for drug discovery. Expert Opin Drug Discov. 2015;10:651-69.

43. Borden MA, Streeter JE, Sirsi SR, Dayton PA. In vivo demonstration of cancer molecular imaging with ultrasound radiation force and buried-ligand microbubbles. Mol Imaging. 2013;12:357-63.

44. Ma J, Xu CS, Gao F, Chen M, Li F, Du LF. Diagnostic and therapeutic research on ultrasound microbubble/nanobubble contrast agents (Review). Mol Med Rep. 2015;12:4022-8.
45. Perera RH, Wu H, Peiris P, Hernandez C, Burke A, Zhang H, Exner AA. Improving performance of nanoscale ultrasound contrast agents using N,N-diethylacrylamide stabilization. Nanomedicine. 2017;13:59-67.

46. Abou-Elkacem L, Wilson KE, Johnson SM, Chowdhury SM, Bachawal S, Hackel BJ, Tian L, Willmann JK. Ultrasound molecular imaging of the breast cancer neovasculature using engineered fibronectin scaffold ligands: a novel class of targeted contrast ultrasound agent. Theranostics. 2016;6:1740-52.

47. Zhou Y, Gu H, Xu Y, Li F, Kuang S, Wang Z, Zhou X, Ma H, Li P, Zheng Y, et al. Targeted antiangiogenesis gene therapy using targeted cationic microbubbles conjugated with CD105 antibody compared with untargeted cationic and neutral microbubbles. Theranostics. 2015;5:399-417.

\section{Submit your next manuscript to BioMed Central and we will help you at every step:}

- We accept pre-submission inquiries

- Our selector tool helps you to find the most relevant journal

- We provide round the clock customer support

- Convenient online submission

- Thorough peer review

- Inclusion in PubMed and all major indexing services

- Maximum visibility for your research

Submit your manuscript at www.biomedcentral com/submit 